\title{
Erratum to: Hypofractionated radiotherapy for localized prostate cancer
}

\author{
Stefan Höcht ${ }^{1} \cdot$ Daniel M. Aebersold ${ }^{2}$ - Clemens Albrecht ${ }^{3} \cdot$ Dirk Böhmer $^{4} \cdot$ Michael Flentje $^{5}$ Ute Ganswindt $^{6}$. \\ Tobias Hölscher $^{7}$ - Thomas Martin ${ }^{8}$ Felix Sedlmayer' $\cdot$ Frederik Wenz $^{10}$ - Daniel Zips ${ }^{11} \cdot$ Thomas Wiegel $^{12}$
}

Published online: 17 October 2016

(C) Springer-Verlag Berlin Heidelberg 2016

\section{Erratum to:}

Strahlenther Onkol 2016192

DOI: $10.1007 / \mathrm{s} 00066-016-1041-5$

Unfortunately, there was a mistake in the title of the original publication.

The correct title reads as follows:

Hypofractionated radiotherapy for localized prostate cancer

We apologize for any inconveniences caused.

The online version of the original article can be found under http://dx.doi.org/10.1007/s00066-016-1041-5.

Stefan Höcht

stefan.hoecht@googlemail.com

1 Xcare Gruppe, Radiologie, Nuklearmedizin und Strahlentherapie, Hubert-Schreiner-Straße 1, 66740 Saarlouis, Germany

2 Universitätsklinik für Radio-Onkologie, Inselspital, University of Bern, Bern, Switzerland

3 Klinik für Radioonkologie und Gemeinschaftspraxis für Strahlentherapie, Klinikum Nürnberg Nord, Universitätsklinikum der Paracelsus Medizinischen Privatuniversität, Nuremberg, Germany

4 Klinik für Radioonkologie und Strahlentherapie, Charité Universitätsmedizin, Berlin, Germany

5 Klinik und Poliklinik für Strahlentherapie, Universitätsklinikum Würzburg, Würzburg, Germany
$6 \quad$ Klinik und Poliklinik für Strahlentherapie und Radioonkologie, Ludwig-Maximilians-Universität München, Munich, Germany

$7 \quad$ Klinik und Poliklinik für Strahlentherapie und Radioonkologie, Universitätsklinikum Carl Gustav Carus, Technische Universität Dresden, Dresden, Germany

8 Klinik für Strahlentherapie und Radioonkologie, Klinikum Bremen-Mitte, Bremen, Germany

9 Universitätsklinik für Radiotherapie und Radio-Onkologie, Landeskrankenhaus, Universitätsklinikum der Paracelsus Medizinischen Privatuniversität, Salzburg, Austria

10 Klinik für Strahlentherapie und Radioonkologie, Universitätsmedizin Mannheim, Universität Heidelberg, Mannheim, Germany

11 Universitätsklinik für Radioonkologie, Universitätsklinikum Tübingen, Tübingen, Germany

12 Abteilung Strahlentherapie, Universitätsklinikum Ulm, Ulm, Germany 